\title{
Dependence of Oxygenation Temperature on Critical Temperature and Current for $\mathrm{Tl}_{2} \mathrm{Ba}_{2} \mathrm{Ca}_{2} \mathrm{Cu}_{3} \mathrm{O}_{x}$
}

\author{
M. Giebultowski*, W.M. Woch, R. Zalecki, J. Michalik, M. Kowalik, \\ J. NiEWOLSKI AND Ł. GONDEK \\ AGH University of Science and Technology, Solid State Physics Dept., \\ Faculty of Science and Applied Computer Science, Mickiewicza 30 30-059 Kraków Poland
}

\begin{abstract}
Thallium based $\mathrm{Tl}_{2} \mathrm{Ba}_{2} \mathrm{Ca}_{2} \mathrm{Cu}_{3} \mathrm{O}_{x}$ superconductors were synthesized in double silver foil at $880{ }^{\circ} \mathrm{C}$ for $30 \mathrm{~min}$ under flowing oxygen gas. Additional oxygenations, each lasting $20 \mathrm{~h}$, were performed at temperatures varying from $700{ }^{\circ} \mathrm{C}$ to $820^{\circ} \mathrm{C}$ to increase the critical temperature and the critical current of the superconductor. Optimal temperature of the oxygenation was of $760^{\circ} \mathrm{C}$.
\end{abstract}

DOI: 10.12693/APhysPolA.137.803

PACS/topics: thallium, cuprate, critical current, critical temperature

\section{Introduction}

Thallium-based superconductors are known from having the highest critical temperatures among cuprates although their properties, especially in the range of transition to the superconducting state, are still poorly recognised. These superconductors, particularly these in the form of thin layers due to their critical temperatures higher than the boiling point of liquid methane (about $112 \mathrm{~K}$ ), can be used for current transmission lines combined with the liquid natural gas transmission lines cooled with this gas.

The case of Tl-2223 compound was previously studied by Kaneko [1] and Nabatame [2]. In the former work (encapsulated or not) Tl-2223 has been heated at $760^{\circ} \mathrm{C}$ in various times, achieving $127 \mathrm{~K}$ of zero resistance transition temperature. In the latter work thin films of Tl-2223 on $\mathrm{MgO}$ substrate have been studied. SEM images of the film surface were confronted with critical current density and heating conditions.

Zalecki et al. [3] studied bulk Tl-2223 sintering in silver foil [4] consisted of two steps for $0.2 \mathrm{~h}$ at $925^{\circ} \mathrm{C}$, and for $10 \mathrm{~h}$ at $910^{\circ} \mathrm{C}$. These steps corresponded, respectively, to the synthesis and the oxygenation steps. There, from AC magnetic susceptibility $T_{c}$ was determined as the critical temperature of separated polycrystalline grain.

Thallium based superconductors in the 2223 system after short (30 min) synthesis in oxygen atmosphere showed low critical temperature of $107.1 \mathrm{~K}$ and current of $37.5 \mathrm{~A} / \mathrm{cm}^{2}$. Thermal treatment in oxygen atmosphere can considerably change properties of a thallium cuprate. Possible processes that might be involved are oxygen interpolation [5], thallium loss [6], and change of chemical

\footnotetext{
* corresponding author; e-mail: marek.giebultowski@fis.agh.edu.pl
}

content [7]. Oxygenation is carried out according to temperature versus time relation.

In this work, we present a detailed study of the dependence of critical temperature and critical current density on the oxygenation conditions of thallium based superconductors.

\section{Experimental}

To obtain $\mathrm{BaCuO}_{2}$ and $\mathrm{Ca}_{2} \mathrm{CuO}_{3}$ precursors, the appropriate amounts of the starting powders were sintered at $840^{\circ} \mathrm{C}$ for $24 \mathrm{~h}$, and then at $940^{\circ} \mathrm{C}$ for $48 \mathrm{~h}$. Next, the materials were reground and placed into furnace for $48 \mathrm{~h}$ at $940^{\circ} \mathrm{C}$. To obtain the Tl-2223 superconductor, appropriate amounts of $\mathrm{BaCuO}_{2}, \mathrm{Ca}_{2} \mathrm{CuO}_{3}$, and $\mathrm{Tl}_{2} \mathrm{O}_{3}$ were ground and pressed into pellets which were wrapped in silver foil. The pellets were heated at $880^{\circ} \mathrm{C}$ for $30 \mathrm{~min}$ in a furnace in flowing oxygen gas. The samples were cut into pieces, and they were oxygenated for $20 \mathrm{~h}$ at various temperatures. For the pieces of the first sample (sample A) the oxygenation temperatures were $700-760^{\circ} \mathrm{C}$. For the other sample (sample B) the oxygenation temperatures were $760-820^{\circ} \mathrm{C}$. The AC susceptibility measurements were done using mutual inductance bridge technique at the $\mathrm{AC}$ magnetic field ranging from 0.022 Oe to 10.9 Oe. A Stanford SR 830 lock-in nanovoltmeter was serving as a source of the AC current for the primary coil and as the nanovoltmeter of the bridge. The temperature was measured with the accuracy of $0.05 \mathrm{~K}$ by a chromelgold- $0.07 \%$ iron thermocouple working with a Lake Shore temperature controller. The samples investigated had shapes of a flat cuboid and the magnetic field direction was parallel to their longest edge. The working frequency was set to $189 \mathrm{~Hz}$. The X-ray powder diffraction (XRD) was performed with Empyrean Panalytical diffractometer $\left(\mathrm{CuK}_{\alpha}\right)$ at $300 \mathrm{~K}$ in $2 \theta$ range from $4^{\circ}$ to $80^{\circ}$. SEM images were obtained with JEOL 5900LV apparatus. 


\section{Results and discussion}

The XRD pattern of the sample A oxygenated at $760{ }^{\circ} \mathrm{C}$ is shown in Fig. 1 . The majority phase of this sample is the Tl-2223 superconductor. The minority phases are residuals of $\mathrm{BaCO}_{3}, \mathrm{BaCuO}_{2}, \mathrm{CaO}$ and $\mathrm{CuO}$, which are not superconducting. The microstructure of this sample was observed in SEM, and the image is shown in Fig. 2. Flat crystals with typical sizes $30 \times 3 \mu \mathrm{m}$ and dark spaces between them in the microstructure are visible. The crystals contact each other and often penetrate the neighbours. No preferred direction along which the crystals are oriented can be distinguished.

The dispersive and absorption parts of AC susceptibility of the samples after the oxygenation process are shown in Fig. 3a and 3b, respectively. Both the intraand inter-grain critical temperatures of the samples were determined from the dispersive parts of AC susceptibility by the method described in [8]. The results are shown in Fig. 4. Additionally, Fig. 4 shows the critical temperatures as fitted parameters of (2). The intra-grain critical temperature raises with increasing oxygenation temperature in the whole range. Both, the inter-grain critical temperature and the critical temperature as fitting parameter have local minimum for the sample oxygenated at $780^{\circ} \mathrm{C}$. The highest intra-grain critical temperature was achieved for the $760^{\circ} \mathrm{C}$ oxygenated sample, however, the highest inter-grain critical temperature and the critical temperature from fitting has the sample oxygenated at $800^{\circ} \mathrm{C}$. The critical current value for given temperature was obtained from the Bean formula [9]:

$$
j_{c}=\frac{2 H_{\mathrm{AC}}}{d},
$$

where $H_{\mathrm{AC}}$ is the $\mathrm{AC}$ magnetic field amplitude, and $d$ is the sample thickness. The critical current calculation for the sample $\mathrm{A}$ and $\mathrm{B}$ oxygenated at temperatures ranging from $700^{\circ} \mathrm{C}$ to $800^{\circ} \mathrm{C}$ are shown in Fig. 5. For high temperature superconductors (HTS), in order to describe

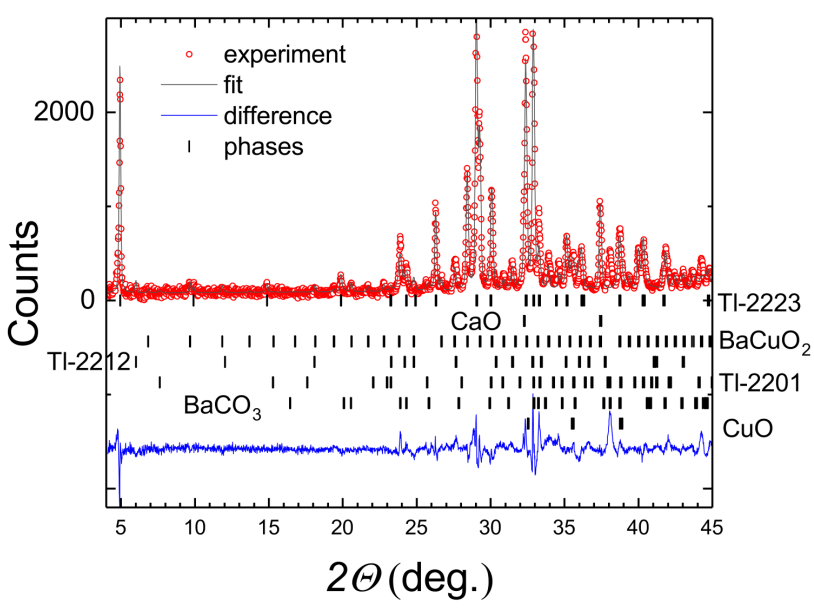

Fig. 1. XRD pattern of the sample A oxygenated at $760{ }^{\circ} \mathrm{C}$.

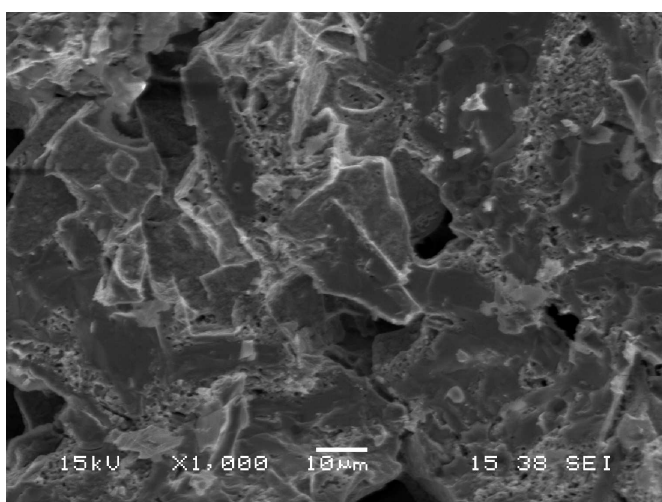

Fig. 2. SEM image of the sample A oxygenation at $760{ }^{\circ} \mathrm{C}$.
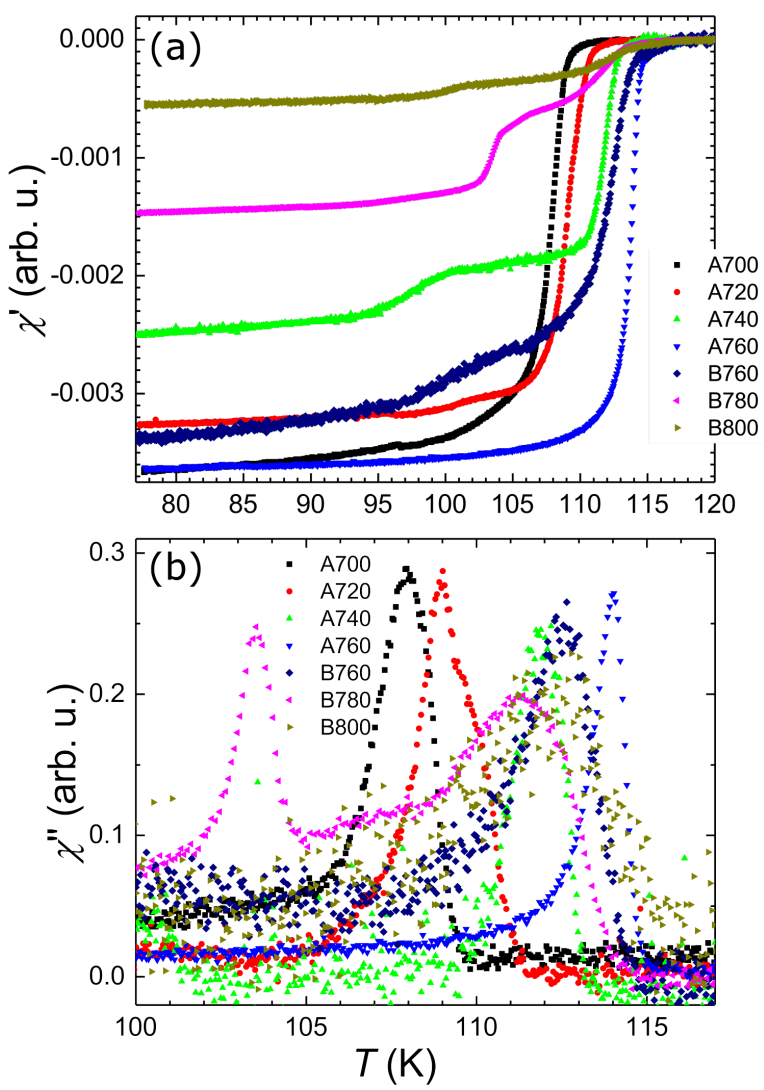

Fig. 3. Dispersive (a), and absorption (b) parts of the AC susceptibility for samples heated at $700-800^{\circ} \mathrm{C}$.

the dependence of the critical current on temperature one can used the Ginzburg-Landau strong coupling limit approach [10, 11], which is expressed by:

$$
j_{c}=j_{c 0}\left(1-\frac{T}{T_{c}}\right)^{n},
$$

where $j_{c 0}$ can be interpreted as critical current at $0 \mathrm{~K}$, $T_{c}$ is the critical temperature, and $n$ is the exponent, which characterises pinning strength. Above Eq. (2) that was used to fit temperature dependence of critical current 


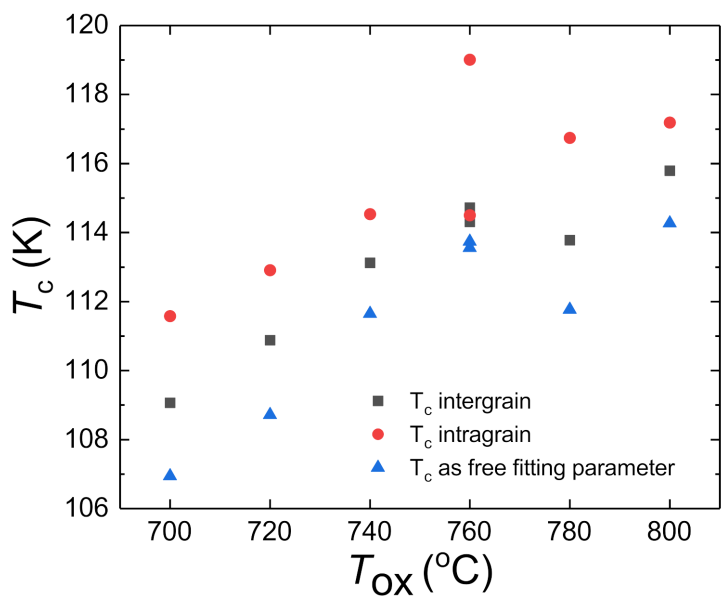

Fig. 4. The values of critical temperature for samples oxygenated in the range from $700^{\circ} \mathrm{C}$ to $800^{\circ} \mathrm{C}$.

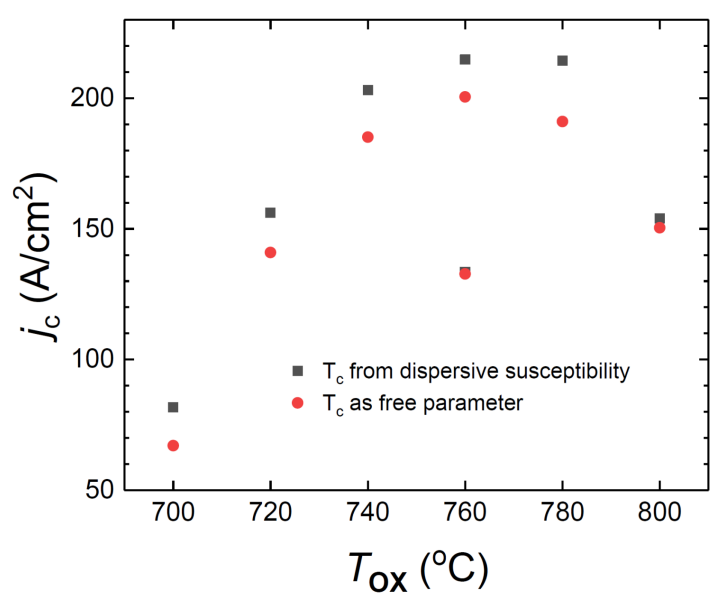

Fig. 5. The critical currents for samples oxygenated at temperatures from $700^{\circ} \mathrm{C}$ to $800^{\circ} \mathrm{C}$.

density, it relates to two cases. In the first case there are two fit parameters: $j_{c 0}$ and $n$, but the third parameter $T_{c}$ is taken from experiment as the inter-grain critical temperature (see Fig. 6a). In the second case three parameters are taken in fitting procedure (see Fig. 6b). Fit parameters are collected in Table I. Taking advantage of the all fitted parameters the critical current densities at the liquid nitrogen temperature were calculated for all the oxygenated samples, and they are shown in Fig. 5. To derive the critical current it was assumed that the value of inter-grain critical temperature is taken from the experiment (black squares in Fig. 5), or $T_{c}$ is treated as a free-fit parameter (red circles in Fig. 5). It can be observed that the critical current density reaches the highest value for both variants.

Strong pinning regime typical for HTS [12] was found to be the case for the most of the studied samples because exponents $n$ were greater than unity. The samples exhibited a vortex glass structure. Only samples A700 and A720 have $n$ lower or greater than unity, depending on fitting procedure details.
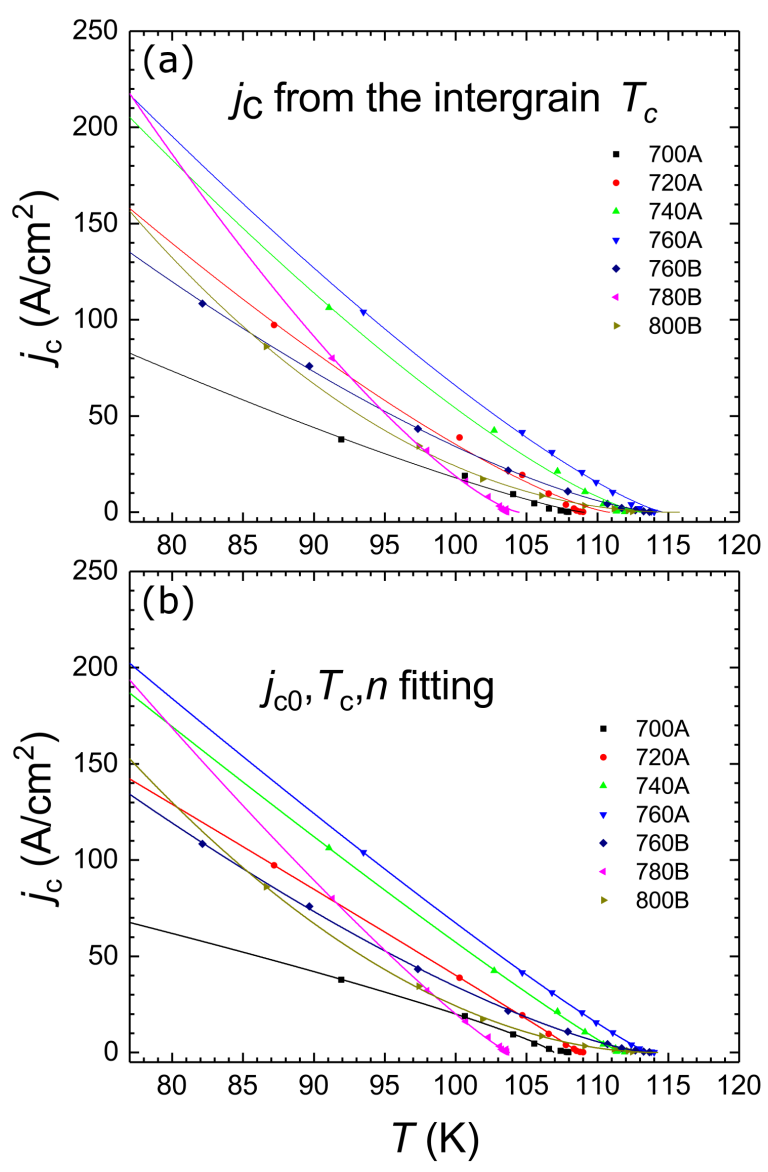

Fig. 6. The critical current versus temperature for samples oxygenated at $700^{\circ} \mathrm{C}$ to $800^{\circ} \mathrm{C}$. (a) $T_{c}$ as intergrain temperature, and (b) $T_{c}$ is free fitting parameter.

Parameters of $j_{c}$ vs. $T$ curves.

TABLE I

\begin{tabular}{c|c|c|c|c}
\hline \hline Sample & $\begin{array}{c}j_{c}\left[\mathrm{~A} / \mathrm{cm}^{2}\right] \\
\text { with } 77.3 \mathrm{~K}\end{array}$ & $\begin{array}{c}j_{c 0} \\
{\left[\mathrm{~A} / \mathrm{cm}^{2}\right]}\end{array}$ & $T_{c}[\mathrm{~K}]$ & $n[1]$ \\
\hline \multicolumn{5}{c}{$j_{c}$ from inter-grain temperature } \\
\hline A700 & 82 & 360 & 109.07 & 1.21 \\
A720 & 156 & 750 & 110.88 & 1.32 \\
A740 & 203 & 920 & 113.12 & 1.32 \\
A760 & 215 & 890 & 114.72 & 1.27 \\
B760 & 134 & 675 & 114.31 & 1.437 \\
B780 & 214 & 1330 & 104.50 & 1.35 \\
B800 & 154 & 1540 & 115.79 & 2.09 \\
\hline \multicolumn{5}{|c}{ Three fitting parameters } \\
\hline A700 & 67 & 195 & 106.9 & 0.83 \\
A720 & 141 & 475 & 108.72 & 0.979 \\
A740 & 185 & 660 & 111.65 & 1.08 \\
A760 & 200 & 711 & 113.56 & 1.109 \\
B760 & 133 & 640 & 113.74 & 1.39 \\
B780 & 191 & 930 & $103.8,111.8$ & 1.16 \\
B800 & 150 & 1310 & 114.27 & 1.92
\end{tabular}


Two oxygenations performed at $760{ }^{\circ} \mathrm{C}$ for $\mathrm{A}$ and $\mathrm{B}$ series indicate different critical temperatures and critical currents. Thus, $T_{c}$ and $j_{c}$ determinations made here should be treated as qualitative.

Our study showed that oxygenation increases both the critical temperature and the critical current of the material. An exception was oxygenation at the highest temperature used $\left(820^{\circ} \mathrm{C}\right)$ where the sample lost its superconductivity related diamagnetism or its critical temperature was lower than $77 \mathrm{~K}$.

\section{Conclusions}

The thallium based $\mathrm{Tl}_{2} \mathrm{Ba}_{2} \mathrm{Ca}_{2} \mathrm{Cu}_{3} \mathrm{O}_{x}$ superconductor compounds oxygenated at temperatures from $700{ }^{\circ} \mathrm{C}$ to $820^{\circ} \mathrm{C}$ for $20 \mathrm{~h}$ were obtained. Maximal $j_{c}$ has been achieved for A760. The inter-grain, free fitting parameter $T_{c}$ was able to raise with the oxygenation temperature up to B800. The highest intra-grain temperature was for A760.

\section{References}

[1] T. Kaneko, H Yamauchi, S. Tanaka, Physica $C$ 178, 377 (1991).

[2] T. Nabatame, Y. Saito, K. Aihara, T. Kamo, Jpn. J. Appl. Phys. 29, L1813 (1990).
[3] R. Zalecki, W.M. Woch, A. Kołodziejczyk, W.T. Konig, G. Gritzner, Acta Phys. Pol. A 126 4A, A-133-136, (2014).

[4] M. Mair, W.T. Konig, G. Gritzner, Supercond. Sci. Technol. 8, 894 (1995).

[5] R.S. Liu, P.P. Edwards in: Thallium-based High Temperature Superconductors, Eds. A.M. Hermann, J.V Jakhmi, Marcel Dekker Inc., New York, Basel, Hong Kong 1994, p. 332.

[6] K.H. Sandhage, P.K. Gallagher in Thallium-based High Temperature Superconductors, Eds. A.M. Hermann, J.V. Jakhmi, Marcel Dekker Inc., New York, Basel, Hong Kong 1994, p.393.

[7] E. Ruckenstein, C.T. Cheung, J. Matter. Res. 4, 1116 (1989)

[8] M. Kowalik, R. Zalecki, W.M. Woch, W. Tokarz, J. Niewolski, Ł. Gondek, J. Supercond. Nov. Magn. 30, 2387 (2017).

[9] C.P. Bean, Phys. Rev. Lett. 8, 250 (1962).

[10] J.R. Clem, B. Bumble, S.I. Raider, W.J. Gallagher, Y.C. Shih, Phys. Rev. B 35, 6637 (1987).

[11] W.M. Woch, R. Zalecki, A. Kołodziejczyk, H. Sudra, G. Gritzner, Supercond. Sci. Technol. 21, 085002 (2008).

[12] W.M. Woch, W. Tokarz, R. Zalecki, A. Kołodziejczyk, C. Deinhofer, G. Gritzner, Supercond. Sci. Technol. 23, 025004 (2010). 